\title{
Estimating the risk of conversion from video-assisted thoracoscopic lung surgery to thoracotomy-a systematic review and meta-analysis
}

\author{
Alexandra D. Power ${ }^{1}$, Robert E. Merritt ${ }^{1}$, Mahmoud Abdel-Rasoul ${ }^{2}$, Susan D. Moffatt-Bruce ${ }^{1}$, \\ Desmond M. D'Souza ${ }^{1}$, Peter J. Kneuertz ${ }^{1}$ \\ ${ }^{1}$ Division of Thoracic Surgery, Department of Surgery, The Ohio State University Wexner Medical Center, Columbus, OH, USA; ${ }^{2}$ Center for \\ Biostatistics, The Ohio State University College of Medicine, Columbus, OH, USA \\ Contributions: (I) Conception and design: AD Power, PJ Kneuertz; (II) Administrative support: RE Merritt, SD Moffatt-Bruce; (III) Provision of study \\ materials and patients: All authors; (IV) Collection and assembly of data: AD Power, PJ Kneuertz; (V) Data analysis and interpretation: AD Power, PJ \\ Kneuertz, M Abdel-Rasoul; (VI) Manuscript writing: All Authors; (VII) Final approval of manuscript: All Authors. \\ Correspondence to: Peter J. Kneuertz, MD. Division of Thoracic Surgery, The Ohio State University Wexner Medical Center, 410 West 10 th Avenue, \\ N846a Doan Hall, Columbus, OH 43210, USA. Email: Peter.Kneuertz@osumc.edu.
}

Background: Understanding the risk of conversion from video-assisted thoracic surgery (VATS) to thoracotomy is important when considering patient selection and preoperative surgical risk assessment. This review aims to estimate the rate of intraoperative conversions to thoracotomy, predictive factors, and associated outcomes for VATS anatomic lung resections.

Methods: PubMed/MEDLINE and EMBASE were searched systematically in May of 2020. Observational studies examining conversions of VATS anatomic resections to thoracotomy were included. Conversion rates, causes, risk factors, and post-operative outcomes were reviewed and analyzed in aggregate.

Results: Twenty retrospective studies were reviewed, with a total of 72,932 patients undergoing VATS anatomic lung resection. The median conversion rate was 9.6\% (95\% CI: 6.6-13.9\%). Nine studies reported a total of 114 emergency conversions, with a median incidence rate of $1.3 \%$ (95\% CI: 0.6-2.8\%). The most common reasons for thoracotomy were vascular injury/bleeding, difficulty lymph node dissection, and adhesions, accounting for $27.9 \%, 26.2 \%$ and $19 \%$ of conversions, respectively. Risk factors for conversion varied, but frequently included nodal disease, large tumors, and induction therapy. The risk of complications (OR 2.06; 95\% CI: 1.77-2.40) and mortality (OR 4.11; 95\% CI: 1.59-10.61) were significantly increased following conversions. There was also a significant increase in chest tube duration and length of stay following conversion.

Conclusions: The risk of conversion to thoracotomy may be as high as one in ten patients undergoing VATS anatomic lung resections, but may vary significantly based on patient selection. Although emergent conversions are rare, the need for thoracotomy may significantly increase postoperative morbidity and mortality.

Keywords: Video-assisted thoracoscopic surgery (VATS); lung resection; lobectomy; conversion; thoracotomy; outcomes

Submitted Sep 23, 2020. Accepted for publication Dec 03, 2020.

doi: $10.21037 /$ jtd-20-2950

View this article at: http://dx.doi.org/10.21037/jtd-20-2950 


\section{Introduction}

Video-assisted thoracoscopic surgery (VATS) has become the most common minimal invasive approach for anatomic lung resection of early stage lung cancer worldwide (1). VATS allows for smaller incisions with fewer chest wall trauma and impact on respiratory mechanics, which has been shown to result in shorter hospital stays, lower rates of complications, and less postoperative pain when compared with thoracotomy $(2,3)$. As the application for VATS expands, one of the concerns often raised is the risk of conversion to an open procedure and possible complications of conversion.

The risk assessment of a conversion and potential associated perioperative complications is important for surgeons with regards to patient selection for VATS and preoperative counseling. These preoperative considerations are critical, especially for patients with marginal pulmonary function at baseline who would be considered high risk for thoracotomy (4). Although there are numerous reports on VATS conversions in the literature, the actual risk and associated outcomes remain ill-defined as they vary greatly between studies, and few studies have reported on emergency conversions (5-13). Several recent studies have sought to identify predictors of conversions, which present a diverse number of cause and risk factors for conversions $(6,9,11,12,14-21)$. Outcomes of VATS operations converted to thoracotomy have also been inconsistent, with some studies showing similar perioperative outcomes to VATS, and others have found increased morbidity and mortality $(6,9,11,13-15,19-22)$.

We conducted a systematic review and meta-analysis of the existing literature on conversions of VATS to thoracotomy for anatomic lung resections in order to (I) define the incidence and reason for VATS conversions (II) summarize risk factors, and (III) assess outcomes associated with VATS conversion as compared to completed VATS procedures. We present the following article in accordance with the PRISMA reporting checklist (available at http:// dx.doi.org/10.21037/jtd-20-2950).

\section{Methods}

\section{Literature search strategy}

We conducted a systematic review of the existing literature using PubMed/MEDLINE and EMBASE databases using the PRISMA standards (23). A MeSH heading was used to perform the database search that included the following search term: "VATS" OR "thoracoscopic" AND "conversion" OR "thoracotomy" AND "lung resection". The database was then filtered by most recent. Titles and abstracts were then reviewed. The references of the studies included from the database were then reviewed for additional studies that met inclusion criteria. Studies that met inclusion criteria were found up to May of 2020. The data of last database search was performed in June 2020. Regulatory approval was not required, as this study did not involve the use of human subjects.

\section{Eligibility criteria}

The preferred reporting items for systematic reviews and meta-analysis (PRISMA) diagram in Figure 1 exhibits the literature search process (23). The inclusion criteria for the literature search were studies limited to adult human subjects and published in English that met the following criteria: (I) study design: observational or clinical trial (II) Intervention: anatomic lung resection and evaluating primary planned VATS procedures that were converted to thoracotomy (III) outcome: Cause and/or risk factors for conversion; and/or postoperative morbidity and mortality. Studies were reviewed from dates ranging from 2000 to 2020 to maintain relevance. We excluded case reports, review articles, abstracts, and studies that did not include either cause for conversion or risk factors, or postoperative outcomes comparing VATS conversions to VATS. These criteria were followed in order to limit bias and review data that strictly pertained to VATS conversion outcomes.

\section{Data extraction and critical appraisal}

The literature search was performed by two reviewers (ADP and PJK) and then analyzed for results. PubMed/ MEDLINE and EMBASE databases were searched for articles, and abstracts were reviewed for inclusion and exclusion criteria. Data was then extracted from the studies meeting inclusion criteria and reviewed for variables such as type of study years, type of resection, conversion rate, emergent conversions, reason for conversion, risk factors for conversion, and postoperative outcomes including complications, length of chest tube duration, length of hospital stay and perioperative mortality (in hospital or within 30-days). Total number of cases for each study were recorded. Each study was then analyzed for the above variables and numbers for each variable found in each study were recorded. There was not a review protocol created or 


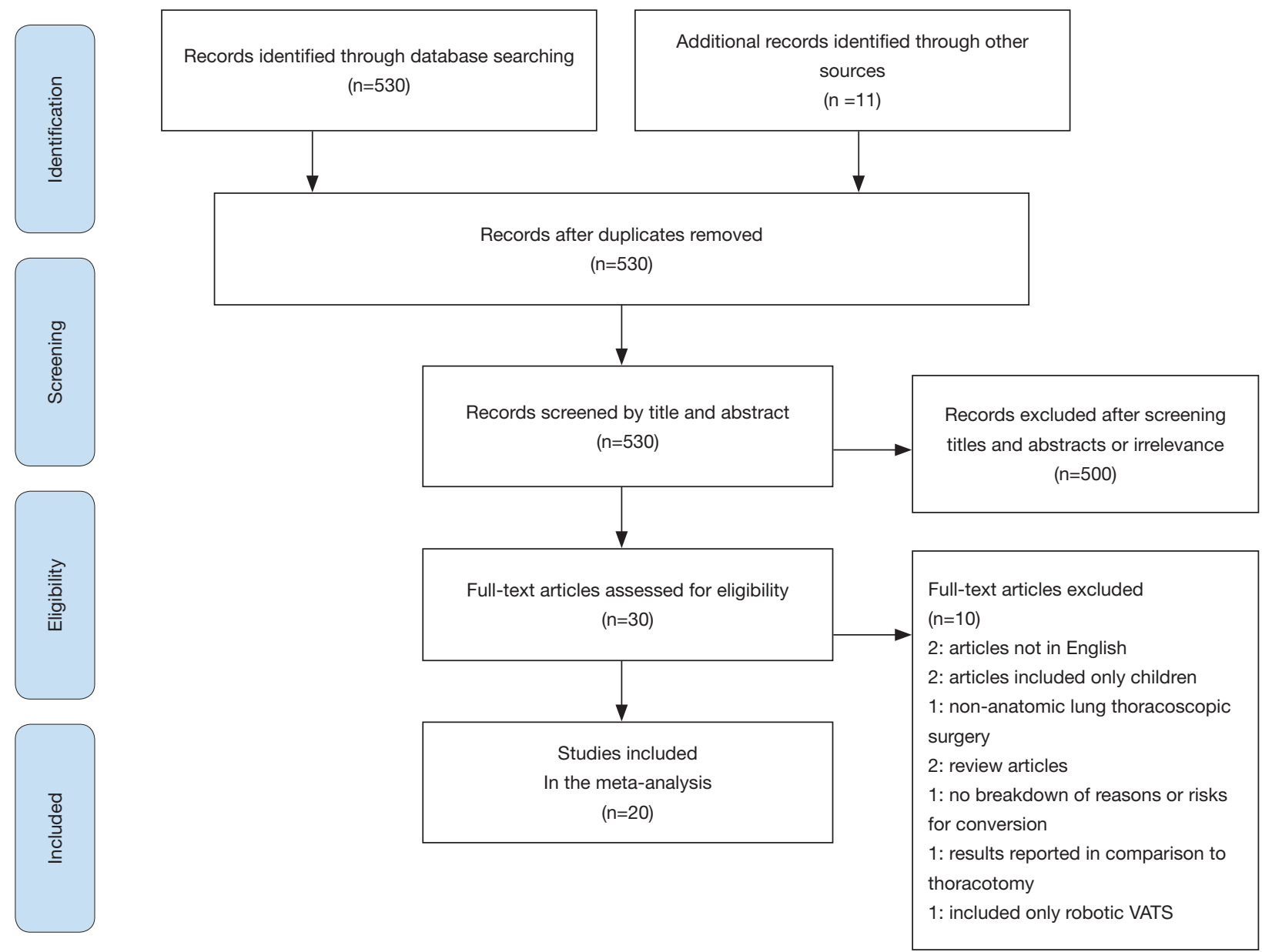

Figure 1 Preferred reporting items for systematic reviews and meta-analysis (PRISMA) outlining selection of study for review and metaanalysis.

filed for this study.

The Downs and Black assessment checklist was used to evaluate the quality of each article included in the review and assess for bias at the study level. The Downs and Black checklist consists of twenty-seven items that evaluate studies on internal validity, external validity, and bias (24). Question 27, which assesses the study power, was excluded from the score. Scores were tallied and defined as low quality articles for a total score between 0-9. Moderate quality articles were those that had a score of 10-18. High quality articles were those that had a score of $\geq 19$. Articles found to be of low quality would plan to be excluded.

\section{Statistical analysis}

First, we performed a random effects meta-analysis to estimate the aggregate frequencies of conversions using a generalized linear mixed model using SAS version 9.4 (SAS Institute, Cary NC) with syntax that was previously described for application in data with rare events (25). Median incidence rates of conversions were expressed as the proportion of conversions with $95 \%$ confidence intervals (CIs). Next, a meta-analysis was performed for perioperative outcomes of VATS conversion as compared to VATS using Review Manager 5.3 (The Nordic Cochrane Centre, Copenhagen, Denmark). Random effects models by methods of Mantel-Haenszel for dichotomous variables and inverse-variance for continuous variables, were used to calculate summary estimates and to adjust for heterogeneity. Aggregate effect measures were expressed as odds rations (ORs) for postoperative events, including minor and major complications, specific complications (pulmonary 
and cardiovascular, and perioperative mortality, and mean differences (MD) for chest tube duration and length of stay, with $95 \%$ CIs. Studies were weighted according to the inverse of the variance their effect estimates. Overall effects were assessed by using the $Z$ test and heterogeneity was tested by using the Cochran's Chi ${ }^{2}$ test. Two sided $\mathrm{P}$ values less than 0.05 were considered statistically significant.

\section{Bias}

There is the potential for bias within the analyzed studies. Reporting bias may occur due to centers having different definitions of complications or how the ultimate cause of conversion is decided. Several studies had varying definitions of cutoffs for tumors size causing conversion and ultimately all decisions to convert are at surgeon discretion. Population bias may also exist as there a few studies that had higher rates of tuberculosis and fungal infections that may lead to increased adhesions (18). This is discussed further in the discussion section.

\section{Results}

The initial search using the MeSH heading yielded 530 results. All articles were screened by title and abstract. Of 30 full text articles that were assessed for eligibility based on inclusion and exclusion criteria, 20 studies met criteria to be included in this review and meta-analysis (Figure 1). All studies were retrospective observational studies, including 18 studies with institutional data and 2 database studies, which are summarized in Table 1. Downs and Black assessment was performed for each article with scores ranging from 15-21, including 8 studies achieving a high quality mark, and 12 studies deemed moderate quality. Thirteen studies included lobectomy cases while only 8 studies evaluated conversions in patients undergoing VATS with any type of anatomic lung resection.

\section{Incidence and reason for conversions}

The frequency of VATS conversions ranged from 1-43\% between studies (Table 1). A total of 7,427 conversions during VATS anatomical lung resections were reported, compiling a weighted aggregate median incidence was 9.6\% (95\% CI: $6.6-13.9 \%$ ). Nine studies reported a total 114 emergency conversions, with a median incidence rate of $1.3 \%$ (95\% CI: 0.6-2.8\%). The reasons for conversions are reported in Table 2. The most common intraoperative reasons for conversions were vascular injury or bleeding (27.9\%; 95\% CI: 24.8-31.1\%), difficult lymph node dissection (26.2\%; 95\% CI: $17.3-37.5 \%)$ and adhesions (19\%; 95\% CI: 13-26.9\%). Less frequent reported causes for conversion included tumor size/location, anatomy or body habitus, incomplete fissures, problems with single lung ventilation or oxygenation, and other technical problems.

\section{Risk factors for conversion}

Thirteen studies evaluated risk factors that may predict a conversion. These analyses varied significantly between studies and included a heterogeneous number of patient, disease, and radiologic data that was available prior to surgery (Table 1). Age was a risk factor listed in 7 studies $(12,14-18,21)$. One study reported age greater than 65 as a significant risk factors for conversion (18). Three of the studies listed age greater than 70 as a significant risk factor $(14,16,21)$. Tumor size was reported as risk factor in five of the studies $(6,11,12,16,19)$. The size of the tumor being of significance for conversion ranged from greater than $1.4 \mathrm{~cm}$ to greater than $4.8 \mathrm{~cm}(11,12,16)$. Male gender was a risk factor found to be significant in four of the reviewed studies $(9,12,14,19)$. Induction therapy was a significant risk factor reported in three studies. Of note, while induction therapy was consistently reviewed as a risk factor in studies, it was considered an exclusion criteria in three studies $(6,12,15,17-19)$. One study by Li et al. of 306 patients found no significant risk factors for conversion when comparing the VATS group to conversion group (26). Other risk factors reported less frequently included history of COPD, history of smoking, surgery performed in a community hospital and increasing BMI (Table 1).

\section{Postoperative outcomes}

Fourteen studies reported postoperative outcomes associated with VATS conversions with mixed results. In aggregate, studies showed an increase in overall complications for VATS conversions as compared to VATS anatomic lung resections with an odds ratio of 2.06 (95\% CI: 1.77-2.40) (Figure 2). Specifically, most studies reported an increase in pulmonary (OR 2.5; 95\% CI: 2.02-3.12), and cardiovascular complications (OR 2.45; 95\% CI: 1.99-3.02) (Figure S1). VATS conversion were associated with a significant increase in postoperative chest tube duration and prolonged length of stay, with an added mean difference of 1.6 (95\% CI: 0.9-2.3) days and 1.8 (95\% CI: 0.7-2.9) days, 


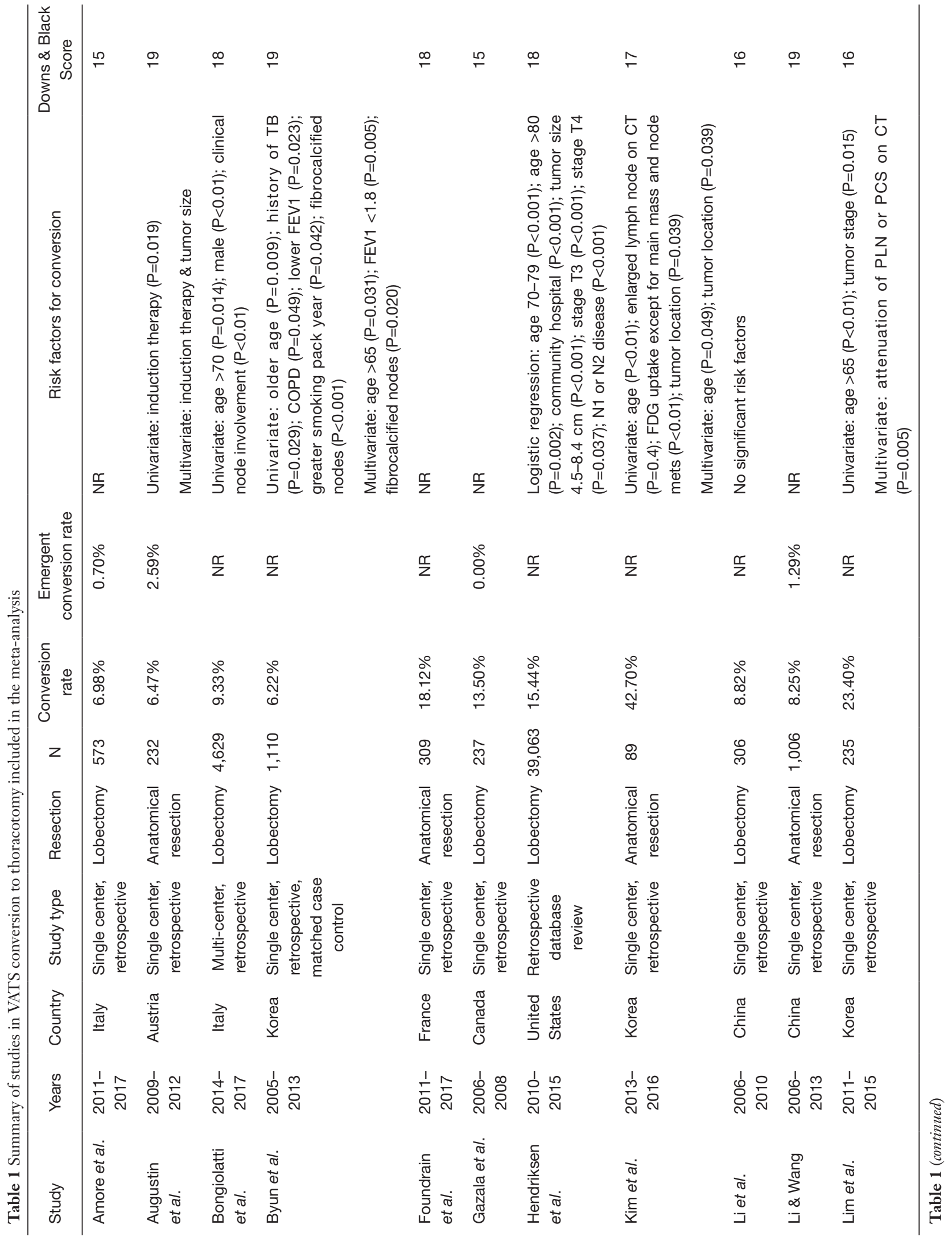




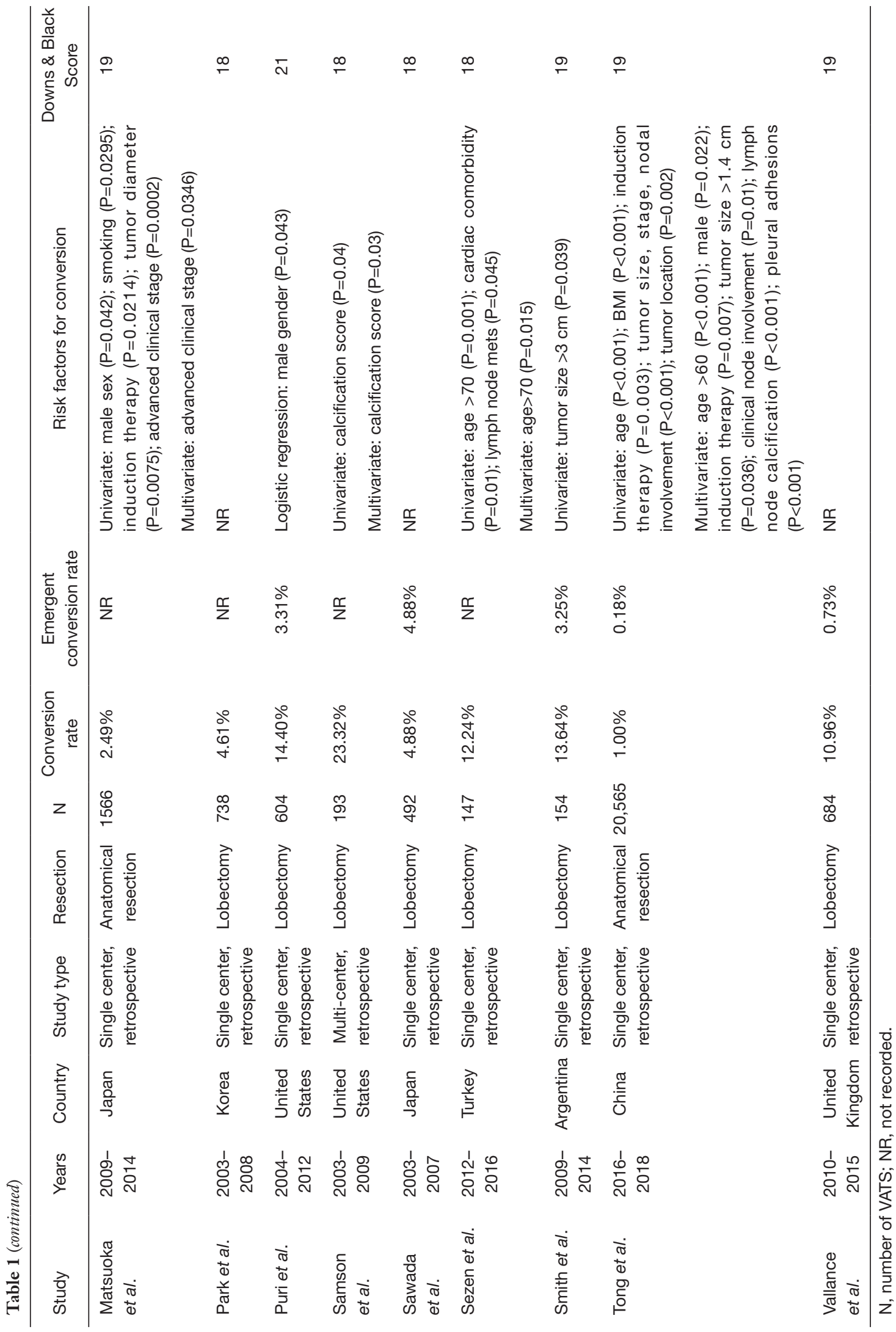


Table 2 Meta-analysis of median incidence rates of VATS conversion by acuity and reason for conversion to thoracotomy

\begin{tabular}{|c|c|c|c|c|}
\hline & Reporting studies (n) & Total number of conversions & Proportion & $95 \% \mathrm{Cl}$ \\
\hline Emergent conversion & 9 & 114 & $1.3 \%$ & $0.6-2.8 \%$ \\
\hline Reason for conversion & 19 & 1,395 & $100 \%$ & \\
\hline Vascular injury/bleeding & & 338 & $27.9 \%$ & $24.8-31.1 \%$ \\
\hline Adhesions & & 291 & $19 \%$ & $13.0-26.9 \%$ \\
\hline Tumor size/location & & 160 & $8.7 \%$ & $5.4-13.7 \%$ \\
\hline Anatomy/body habitus & & 101 & $5 \%$ & $2.7-9.1 \%$ \\
\hline Incomplete fissure & & 76 & $3.3 \%$ & $1.8-6.1 \%$ \\
\hline Other & & 30 & $2.7 \%$ & $1.3-5.5 \%$ \\
\hline
\end{tabular}

VATS, video-assisted thoracic surgery.

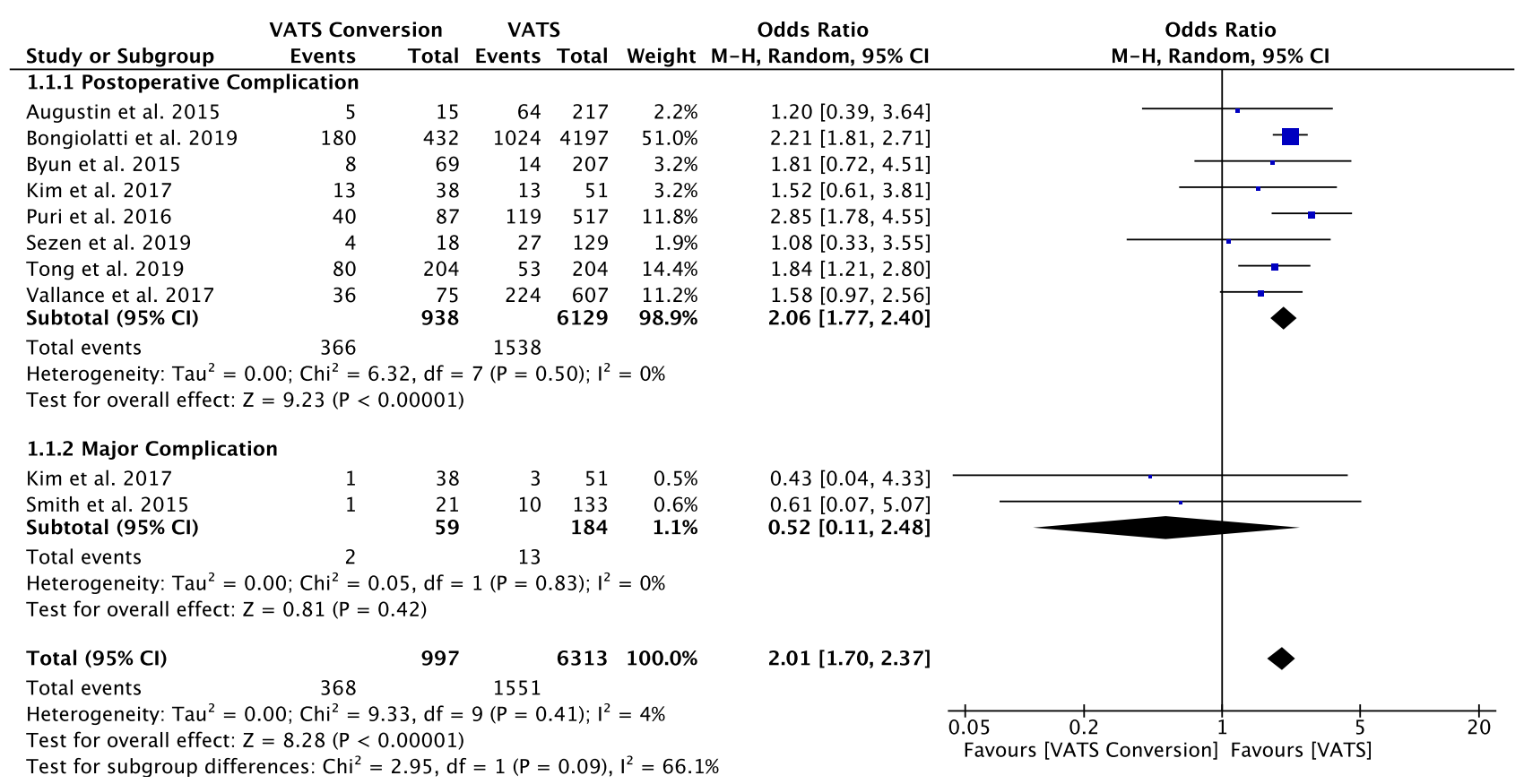

Figure 2 Forrest plot. Association of postoperative complications for VATS conversions versus VATS. VATS, video-assisted thoracic surgery.

respectively (Figure 3). Although few studies reported on major complications (Figure 2), and mortality rates were overall low, VATS conversions were associated with a four times increase odds in early postoperative mortality (OR 4.1; 95\% CI: 1.59-10.61) (Figure 4).

\section{Discussion}

This systematic review and meta-analysis evaluates the risk and associated outcomes of conversions to thoracotomy for planned VATS anatomic lung resection. The feasibility 


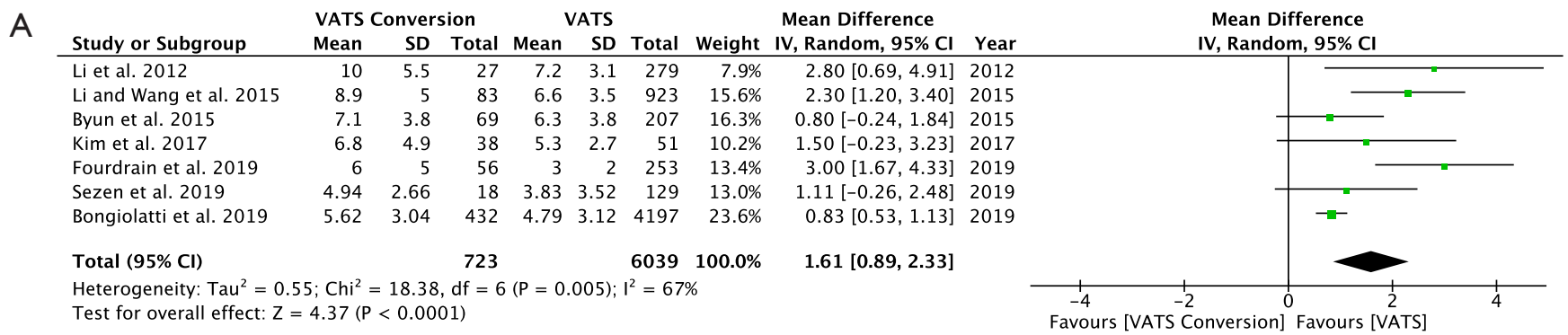

B

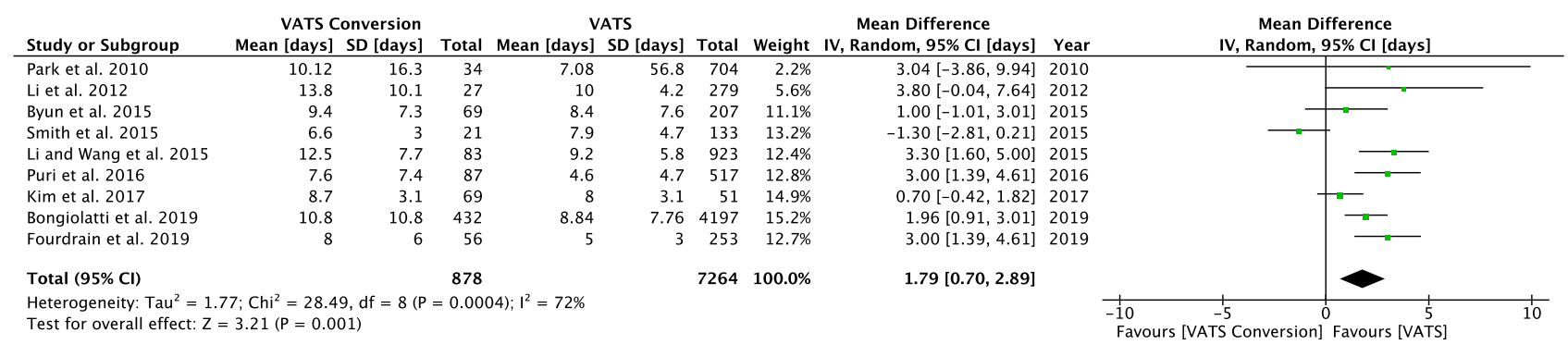

Figure 3 Forrest plots for (A) length chest tube duration and (B) hospital stay associated with VATS conversions. VATS, video-assisted thoracic surgery.

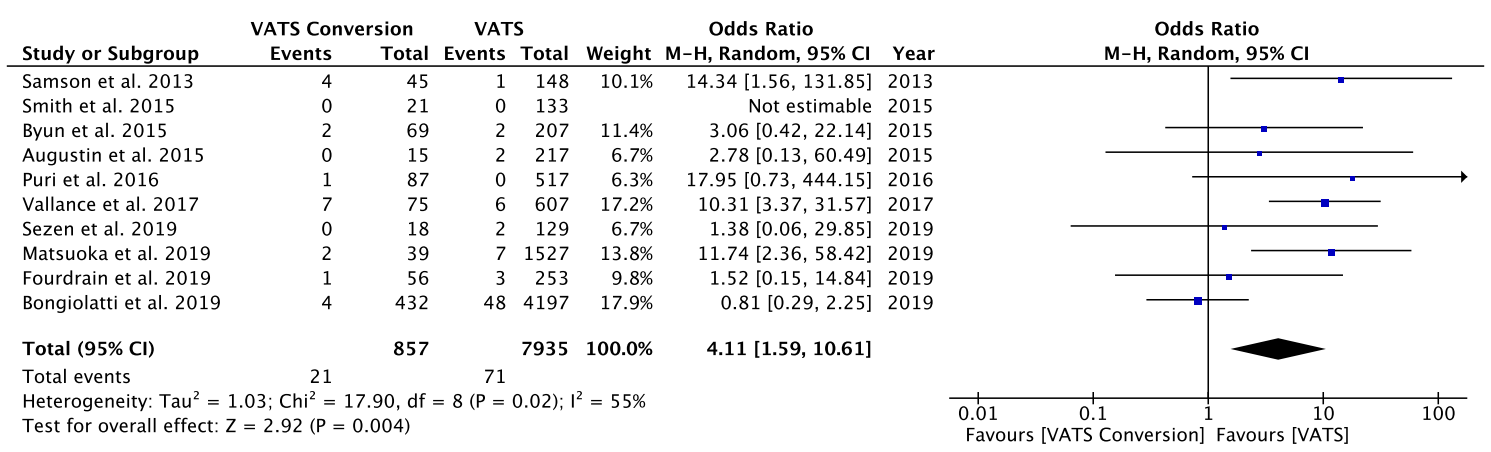

Figure 4 Forrest plot. Association of VATS conversions and mortality. VATS, video-assisted thoracic surgery.

and oncologic efficacy of VATS lobectomy and expedited recovery as compared to thoracotomy has long been established (2). However, technical limitations of the VATS approach have been attributed to the relatively slow adoption over the past two decades. The potential for intraoperative complications that would result in the need for conversion and associated increased morbidity have remained a concern. While multiple recent studies have analyzed conversions in large case series, it has been difficult to estimate the true risk to this current time, because studies have reported a wide range of conversion rates and mixed results regarding morbidity and mortality associated with conversion to thoracotomy.

The first objective of this study was to define the conversion rate and reasons for conversions for VATS anatomical lung resections in the current literature. In a meta-analysis of over seventy-two thousand VATS procedures performed worldwide, we found that the median conversion rate to thoracotomy was just under $10 \%$. While this may seem higher than the conversion rates seen at some expert centers, the range of conversion rates spanned from $1 \%$ to $43 \%$ and were generally higher end of the spectrum in multi-center and population based studies (Table 1). The variations likely reflect differences in patient selection, 
surgeon experience, and operative volume $(12,16,17,21)$. Although vascular injury or bleeding was the most common reason for conversion, emergent conversions were reported as rare events (median incidence $1.3 \%$ ), which speaks to the safety of the VATS approach. In the contrary, most conversions were technical in nature, due to difficult lymph node dissection, tumor size/location, challenging anatomy or adhesion. Clearly, the surgical experience with VATS has shown to affect the conversion rates, and may explain the large variation between studies. Multiple centers have reported on their learning curve with VATS during the initial adoption of this technique. A typical learning curve has been reported to be around 40-60 cases, after which a plateau in operative times for anatomic lung resections can be observed, parallel to a significant decrease in conversion rates (27). The learning curve may also explain some of the variation in conversion rates presented in this review. For example, the study by Kim et al. reported a high conversion rate of $43 \%$ which was significantly higher than most of the other studies examined (17). Kim et al. discusses that their conversion rate could largely be attributed to learning curve. They state a conversion rate of $48 \%$ in 2015 with a decrease to $39 \%$ in 2016 (17). The study by Kim et al. also had the smallest samples sizes which may also indicate decreased case volumes with slower learning curve (17). The study by Samson et al. also reports a slightly higher rate of conversion at $23 \%$ where they too report including their initial learning curve which may contribute to this increase in overall conversion rate (20). The study by Lim et al. also reports a slightly higher rate of conversion at $23.4 \%$, which the authors attribute to a high incidence of tuberculosis and associated anthracofibrosis and adhesions (18). This may make this patient population at a higher risk of conversion at baseline than other studies. Samson et al. also report that their study took place in an area with high rates of histoplasmosis which may lead to increased difficulty of nodal dissection in their patient population and thus contributing to the slightly higher conversion rate (20). However, even beyond the initial learning curves, centers with high volume VATS surgery have demonstrated a continued downward trend of conversions with a growing surgical experience. A study by Puri and colleagues reported a decrease in conversion rate over three-year intervals from $28 \%$ to $15 \%$ to $11 \%$ (9). Notably, one of the most recent and largest institutional reports by Tong et al. reported very low conversion rates, which continued to decrease over a recent three-year span from $1.3 \%$ to $0.8 \%$ (12). It is evident, that with growing experience, VATS surgeons may continue to overcome some of the technical limitation and master difficult anatomic resection for lymph node disease or proximal or large tumors. Not surprisingly, the intraoperative cause for conversion has also been reported to change with increasing VATS experience. Based on a previously suggested simplified system for categorization of VATS conversions, centers who have broadened their operative spectrum beyond the initial learning curve have observed conversions largely due to oncological reasons in patients with larger tumors, lymph node disease and difficult post induction resections (7). The causal spectrum and frequency of conversions in this review indicate that reporting centers of the included studies have likely exceeded their learning curve. Operative strategies have also been developed for managing intraoperative bleeding, which was shown to be the most common cause for conversion in this meta-analysis (Table 2). During VATS, bleeding is typically first controlled with compression, directed application of cautery for bronchial artery or parenchymal bleeding, and pulmonary artery injuries may be repairable by experienced surgeons skilled in VATS by application of clips to smaller branches and preferably by suture in a safe and controlled fashion with proximal vascular control, avoiding the need for thoracotomy (28).

The second objective of this paper was to summarize risk factors for conversions. Here we found the most variation between studies, which examined various patient, disease and radiologic characteristics that may be used to predict the risk of conversion. It is evident that patient and disease factors can significantly affect the risk of a conversion. Lymph node disease or radiographic calcification of intrapulmonary lymph nodes was the most consistent risk factor for conversion (Table 1). Other reported risk factors were advanced age, male gender, large tumor size, and induction therapy (Table 1). However, predictors were studied inconsistently between studies and using different cutoffs. The tumor size associated with increased conversion rates for example varied from $1.4 \mathrm{~cm}$ reported in one study to $4.8 \mathrm{~cm}$ in another $(11,12,16)$. The study by Tong et al. reported tumors greater than $1.4 \mathrm{~cm}$ as a risk factor, but states that $4.9 \%$ of all conversions were sublobar resections, which may explain why a smaller tumor diameter was found to be significant for conversion when compared with other studies (12). Several studies treated induction therapy as an exclusion criterion, while others did not consider it a contraindication for VATS, but found it to be a risk factor for conversion (Table 1). Male gender was also cited as risk factor for conversion in 
several studies $(9,12,14,19)$. Few studies comment on the association between male gender and conversion. Tong et $a l$. found that male patients were older and had higher rates of pleural adhesions, which may have accounted for the relationship of gender and conversions (12). Notably, it has been shown that the use of robotic-assisted thoracoscopic approach for minimally invasive lobectomy, which has seen a rapidly adoption in recent years, may reduce the risk of conversion (3). Hendrickson and colleagues showed that the robotic approach was associated with a significant lower conversion rates as compared to VATS in their propensity score matched analysis of thoracoscopic lobectomy cases in the US National Cancer Database (16).

The third objective of this paper was to determine the outcomes associated with VATS conversions. The results of the individual studies reviewed have been mixed, with some studies attributing increased morbidity from conversions and others do not. In the meta-analysis, we demonstrate that conversion to thoracotomy was associated with a twofold increase in complication rates as compared to VATS completed procedures (Figure 1). In particular, studies reported increase in pulmonary and cardiovascular events after conversions, which may be attributed to the additional chest wall trauma of a thoracotomy and the associated decrease in respiratory mechanics, especially in high-risk patients with baseline marginal pulmonary function (4). Conversion to thoracotomy resulted in an increase in chest tube duration and length of stay in the ranging 1-2 days on average as compared with VATS completed procedures. Overall, these results are comparable to previous studies comparing the outcomes of anatomic resections by VATS versus planned thoracotomy $(2,3)$. This observation is supported by three studies that found no difference between morbidity and mortality between VATS resections that were converted to thoracotomy and those performed with planned thoracotomy $(9,11,17)$. With regards to mortality, the risk following VATS anatomic lung resection was overall low and few studies showing a statistically significant increase. However, with the increased power of this meta-analysis we were able to demonstrate an increase in mortality risk associated with conversion to thoracotomy.

One variable that may be considered for future research that is not discussed as an outcome in the included studies is the degree of patient reported outcomes. Upon literature search, there is no current publication that directly compares patient reported outcomes between VATS and VATS procedures conversion converted to thoracotomy. However, this comparison may be extrapolated form the comparison of VATS with planned thoracotomy. In a systematic review we have recently demonstrated improved symptom recovery and health related quality of life, specifically in the domains of pain and physical functioning in patients undergoing VATS compared to thoracotomy for anatomic lung resection (29).

There are several limitations of our study, which are inherent to any aggregate data analysis. When interpreting the results of our meta-analysis, one needs to keep in mind that the experience and operative volume of surgeon may vary between studies. Several studies have demonstrated a reduction in conversion rates based on operative volume that extends well beyond the initial learning curve. The specific VATS technique and number of ports was also not standardized and may affect the conversion rates. Robotic-assisted thoracoscopic resections were excluded for this reason. Another limitation is that the selection criteria for VATS resection can vary, with some institutions having broader selection than others do, which includes characteristics such as larger or central tumors, lymph node disease, or previous induction therapy. There was reported to be $26 \%$ of cases in this meta-analysis due to lymph node disease. This number may be affected by the varying definitions among centers for VATS. There may also be a degree of cofounding. For example, if a vascular injury occurred due to a difficult lymph node dissection. The cause of conversions due to lymph node dissection may also be affected by the patient populations included in this review. Matsuoka et al. report that 10 out of the 12 patients converted for silicotic lymph nodes had a history of dust inhalation (19). Similarly, studies by Lim et al. and Samson et al. reported higher incidence of TB and histoplasmosis respectively $(18,20)$. Another limitation of the study is that definitions for causes of conversion may vary. For example, there were no clear definitions across studies that specifically described what constituted an anatomic conversion versus what constituted a conversion for lymph node disease. Another limitation to consider in review of the included studies is location of tumor. The location of tumors in conversion groups was reported in 9 studies but only 1 study by Amore et al. linked the location of tumor with actual an actual reason for conversion $(5,6,10,12,15,16,18,20,21)$. The majority of the 9 studies did appear to find higher percentages of tumors in the left upper lobe and right upper lobes in the conversion groups although this was not listed as a significant risk factor for conversion in most $(5,6,10,12,15,16,18,20,21,30)$. Additionally, dissection of central tumors may pose a greater risk of vascular injury 
given the proximity to the pulmonary artery leading to greater numbers of conversion. This may also contribute to overall conversion rate in studies depending on the number of cases performed for central tumors in a given patient population. Publication bias may also have affected the estimation of emergency conversion rates, which are rarely reported in studies. Nonetheless, this systematic review and meta-analysis is important, as it synthesizes the current published data on VATS conversions available thus far so to help advance our professional practice.

\section{Conclusions}

In this systematic review and meta-analysis we estimate the risk of conversion to thoracotomy during VATS anatomic lung resection is approximately $10 \%$. Vascular injury or bleeding, difficult lymph node dissection or adhesions have been consistently reported as the most common reasons for requiring thoracotomy, and conversions are rarely performed emergently. Clinical lymph node disease and calcifications may predict an increased risk of conversions. VATS conversions to thoracotomy are associated with increased risk for postoperative morbidity and mortality, which appear to be similar to conventional outcomes after planned thoracotomy for anatomic lung resections. Our summary of findings can serve as a benchmark for surgeons when comparing their own outcomes with VATS, as well as global reference for future studies.

\section{Acknowledgments}

Funding: None.

\section{Footnote}

Reporting Checklist: The authors have completed the PRISMA reporting checklist. Available at http://dx.doi.org/10.21037/ jtd-20-2950

Conflicts of Interest: All authors have completed the ICMJE uniform disclosure form (available at http://dx.doi. org/10.21037/jtd-20-2950). Dr. REM reports other from Intuitive Surgical, outside the submitted work. Dr. PJK serves as an unpaid editorial board member of fournal of Thoracic Disease from Aug 2020 to Jul 2022. Dr. DMD reports other from Intuitive Surgical, outside the submitted work. The other authors have no conflicts of interest to declare.
Ethical Statement: The authors are accountable for all aspects of the work in ensuring that questions related to the accuracy and integrity of any part of the work are appropriately investigated and resolved.

Open Access Statement: This is an Open Access article distributed in accordance with the Creative Commons Attribution-NonCommercial-NoDerivs 4.0 International License (CC BY-NC-ND 4.0), which permits the noncommercial replication and distribution of the article with the strict proviso that no changes or edits are made and the original work is properly cited (including links to both the formal publication through the relevant DOI and the license). See: https://creativecommons.org/licenses/by-nc-nd/4.0/.

\section{References}

1. Ng CS, MacDonald JK, Gilbert S, et al. Expert consensus statement on optimal approach to lobectomy for non-small cell lung cancer. Innovations (Phila) 2019;14:87-89.

2. Whitson BA, Groth SS, Duval SJ, et al. Surgery for early-stage non-small cell lung cancer: a systematic review of the video-assisted thoracoscopic surgery versus thoracotomy approaches to lobectomy. Ann Thorac Surg 2008;86:2008-16.

3. Kneuertz PJ, Singe E, D'Souza DM, et al. Hospital cost and clinical effectiveness of robotic-assisted versus videoassisted thoracoscopic and open lobectomy: a propensity score-weighted comparison. J Thorac Cardiovasc Surg 2019;157:2018-26.e2.

4. Kneuertz PJ, D'Souza DM, Moffatt-Bruce SD, et al. Robotic lobectomy has the greatest benefit in patients with marginal pulmonary function. J Cardiothorac Surg 2018;13:56.

5. Amore D, Natale DD, Scaramuzzi R, et al. Reasons for conversion during VATS lobectomy: what happens with increased experience. J Vis Surg 2018;4:53.

6. Augustin F, Maier HT, Weissenbacher A, et al. Causes, predictors and consequences of conversion from VATS to open lung lobectomy. Surg Endosc 2016;30:2415-21.

7. Gazala S, Hunt I, Valji A, et al. A method of assessing reasons for conversion during video-assisted thoracoscopic lobectomy. Interact Cardiovasc Thorac Surg 2011;12:962-4.

8. Li Y, Wang J. Analysis of lymph node impact on conversion of complete thoracoscopic lobectomy to open thoracotomy. Thorac Cancer 2015;6:704-8.

9. Puri V, Patel A, Majumder K, et al. Intraoperative 
conversions from video-assisted thoracoscopic surgery lobectomy to open thoracotomy: a study of causes and implications. J Thorac Cardiovasc Surg 2015;149:55-61.

10. Sawada S, Komori E, Yamashita M. Evaluation of videoassisted thoracoscopic surgery lobectomy requiring emergency conversion to thoracotomy. Eur J Cardiothorac Surg 2009;36:487-90.

11. Smith DE, Dietrich A, Nicolas M, et al. Conversion during thoracoscopic lobectomy: related factors and learning curve impact. Updates Surg 2015;67:427-32.

12. Tong C, Li T, Huang C, et al. Risk factors and impact of conversion to thoracotomy from 20565 cases of thoracoscopic surgery. Ann Thorac Surg 2020;109:1522-9.

13. Vallance A, Tcherveniakov P, Bogdan C, et al. The evolution of intraoperative conversion in video assisted thoracoscopic lobectomy. Ann R Coll Surg Engl 2017;99:129-33.

14. Bongiolatti S, Gonfiotti A, Viggiano D, et al. Risk factors and impact of conversion from VATS to open lobectomy: analysis from a national database. Surg Endosc 2019;33:3953-62.

15. Byun CS, Lee S, Kim DJ, et al. Analysis of unexpected conversion to thoracotomy during thoracoscopic lobectomy in lung cancer. Ann Thorac Surg 2015;100:968-73.

16. Hendriksen BS, Hollenbeak CS, Taylor MD, et al. Minimally invasive lobectomy modality and other predictors of conversion to thoracotomy. Innovations (Phila) 2019;14:342-52.

17. Kim SW, Hong JM, Kim D. What is difficult about doing video-assisted thoracic surgery (VATS)? A retrospective study comparing VATS anatomical resection and conversion to thoracotomy for lung cancer in a universitybased hospital. J Thorac Dis 2017;9:3825-31.

18. Lim CG, Shin KM, Lim JS, et al. Predictors of conversion to thoracotomy during video-assisted thoracoscopic surgery lobectomy in lung cancer: additional predictive value of FDG-PET/CT in a tuberculosis endemic region. J Thorac Dis 2017;9:2427-36.

19. Matsuoka K, Yamada T, Matsuoka T, et al. Analysis of conversion to thoracotomy during thoracoscopic lung resection. Asian Cardiovasc Thorac Ann 2019;27:381-87.

20. Samson P, Guitron J, Reed MF, et al. Predictors of conversion to thoracotomy for video-assisted thoracoscopic lobectomy: a retrospective analysis and the influence of computed tomography-based calcification assessment. J
Thorac Cardiovasc Surg 2013;145:1512-8.

21. Sezen CB, Bilen S, Kalafat CE, et al. Unexpected conversion to thoracotomy during thoracoscopic lobectomy: a single center analysis. Gen Thorac Cardiovasc Surg 2019;67:969-75.

22. Fourdrain A, De Dominicis F, Iquille J, et al. Intraoperative conversion during video-assisted thoracoscopy does not constitute a treatment failure. Eur J Cardiothorac Surg 2019;55:660-5.

23. Stewart LA, Clarke M, Rovers M, et al. Preferred reporting items for systematic review and meta-analyses of individual participant data. JAMA 2015;313:1657-65.

24. Downs SH, Black N. The feasibility of creating a checklist for the assessment of the methodological quality both of randomized and non-randomized studies of health care interventions. J Epidemiol Community Health 1998;52:377-84.

25. Stijnen T, Hamza T, Ozdemir P. Random effect metaanalysis of event outcome in the framework of the generalized linear mixed model with applications in sparse data. Stat Med 2010;29:3046-67.

26. Li Y, Wang J, Yang F, et al. Indications for conversion of thoracoscopic to open thoracotomy in video-assisted thoracoscopic lobectomy. ANZ J Surg 2012;82:245-50.

27. Power AD, D'Souza DM, Moffatt-Bruce SD, et al. Defining the learning curve of robotic thoracic surgery: what does it take? Surg Endosc 2019;33:3880-8.

28. Mei J, Pu Q, Liao H, et al. A novel method for troubleshooting vascular injury during anatomic thoracoscopic pulmonary resection without conversion to thoracotomy. Surg Endosc 2013;27:530-7.

29. Singer ES, Kneuertz PJ, Nishimura J, et al. Effect of operative approach on quality of life following anatomic lung cancer resection. J Thorac Dis 2020;12:6913-9.

30. Park JS, Kim HK, Choi YS, et al. Unplanned conversion to thoracotomy during video-assisted thoracic surgery does not compromise the surgical outcome. World J Surg 2011;35:590-5.

Cite this article as: Power AD, Merritt RE, Abdel-Rasoul M, Moffatt-Bruce SD, D’Souza DM, Kneuertz PJ. Estimating the risk of conversion from video-assisted thoracoscopic lung surgery to thoracotomy - a systematic review and meta-analysis. J Thorac Dis 2021;13(2):812-823. doi: 10.21037/jtd-20-2950 
Supplementary

VATS Conversion VATS

Odds Ratio

Odds Ratio

Study or Subgroup Events Total Events

Total Weight M-H, Random, $95 \% \mathrm{Cl}$ Year $\mathrm{M}-\mathrm{H}$, Random, $95 \% \mathrm{CI}$

\begin{tabular}{lrrrrrrr}
\hline 2.1.1 Pulmonary Complication & & & & & & & \\
Samson et al. 2013 & 16 & 45 & 25 & 148 & $2.8 \%$ & $2.71[1.29,5.73]$ & 2013 \\
Byun et al. 2015 & 8 & 69 & 11 & 207 & $1.7 \%$ & $2.34[0.90,6.07]$ & 2015 \\
Puri et al. 2016 & 29 & 87 & 51 & 517 & $5.6 \%$ & $4.57[2.69,7.77]$ & 2016 \\
Vallance et al. 2017 & 44 & 75 & 214 & 607 & $6.6 \%$ & $2.61[1.60,4.25]$ & 2017 \\
Bongiolatti et al. 2019 & 165 & 432 & 879 & 4197 & $36.5 \%$ & $2.33[1.89,2.87]$ & 2019 \\
Sezen et al. 2019 & 8 & 18 & 28 & 129 & $1.5 \%$ & $2.89[1.04,8.00]$ & 2019 \\
Tong et al. 2019 & 78 & 204 & 53 & 204 & $8.9 \%$ & $1.76[1.16,2.69]$ & 2019 \\
Subtotal (95\% Cl) & & $\mathbf{9 3 0}$ & & $\mathbf{6 0 0 9}$ & $\mathbf{6 3 . 6 \%}$ & $\mathbf{2 . 5 1}[\mathbf{2 . 0 2}, \mathbf{3 . 1 2}]$ &
\end{tabular}

Total events $348 \quad 1261$

Heterogeneity: $\mathrm{Tau}^{2}=0.02 ; \mathrm{Chi}^{2}=8.09, \mathrm{df}=6(\mathrm{P}=0.23) ; \mathrm{I}^{2}=26 \%$

Test for overall effect: $Z=8.29(P<0.00001)$

2.1.2 Cardiovascular Complication

Samson et al. 2013

Byun et al. 2015

$\begin{array}{lllll}10 & 45 & 14 & 148 & 2.0 \%\end{array}$

Puri et al. 2016

Vallance et al. 2017

Sezen et al. 2019

Tong et al. 2019

Bongiolatti et al. 2019

Subtotal $(95 \% \mathrm{Cl})$

Total events

$\begin{array}{rrrr}45 & 14 & 148 & 2.0 \% \\ 69 & 2 & 207 & 0.2 \%\end{array}$

$\begin{array}{llll}87 & 55 & 517 & 4.5 \%\end{array}$

$\begin{array}{llll}75 & 38 & 607 & 2.4 \%\end{array}$

$\begin{array}{llll}18 & 6 & 129 & 0.7 \%\end{array}$

$\begin{array}{lll}6 & 129 & 0.7 \% \\ 6 & 204 & 1.5 \%\end{array}$

$405 \quad 4197 \quad 25.1 \%$

$600936.4 \%$

$2.73[1.12,6.68] 2013$

$0.59[0.03,12.47] 2015$

$2.19[1.22,3.95] 2016$

$1.79[0.80,3.99] 2017$

$4.10[0.93,18.12] 2019$

$1.70[0.61,4.77] 2019$

$2.60[2.03,3.35] 2019$

$2.45[1.99,3.02]$

Heterogeneity: $\mathrm{Tau}^{2}=0.00 ; \mathrm{Chi}^{2}=2.80, \mathrm{df}=6(\mathrm{P}=0.83) ; \mathrm{I}^{2}=0 \%$

Test for overall effect: $Z=8.46(P<0.00001)$

Total $(95 \% \mathrm{Cl})$

1860

$12018 \quad 100.0 \%$

Total events

491 1787

Heterogeneity: $\operatorname{Tau}^{2}=0.00 ; \mathrm{Chi}^{2}=10.90, \mathrm{df}=13(\mathrm{P}=0.62) ; \mathrm{I}^{2}=0 \%$

Test for overall effect: $Z=13.95(P<0.00001)$

Test for subgroup differences: $\mathrm{Chi}^{2}=0.02, \mathrm{df}=1(\mathrm{P}=0.88), \mathrm{I}^{2}=0 \%$

$2.44[2.15,2.77]$

Figure S1 Forrest plots for (A) postoperative pulmonary and (B) cardiovascular complication associated with VATS conversions. 